\title{
Universality, Lee-Yang Singularities, and Series Expansions
}

\author{
Gökçe Başar®* \\ Department of Physics and Astronomy, University of North Carolina, Chapel Hill, North Carolina 27599, USA
}

(Received 17 June 2021; accepted 28 September 2021; published 21 October 2021)

\begin{abstract}
We introduce a new way of reconstructing the equation of state of a thermodynamic system near a second-order critical point from a finite set of Taylor coefficients computed away from the critical point. We focus on the Ising universality class ( $\mathbb{Z}_{2}$ symmetry) and show that, in the crossover region of the phase diagram, it is possible to efficiently extract the location of the nearest thermodynamic singularity, the LeeYang edge singularity, from which one can (i) determine the location of the critical point, (ii) constrain the nonuniversal parameters that maps the equation of state to that of the Ising model in the scaling regime, and (iii) numerically evaluate the equation of state in the vicinity of the critical point. This is done by using a combination of Padé resummation and conformal maps. We explicitly demonstrate these ideas in the celebrated Gross-Neveu model.
\end{abstract}

DOI: 10.1103/PhysRevLett.127.171603

Introduction.-In the vicinity of a critical point, the correlation length of a thermodynamic system grows, the underlying microscopic properties become irrelevant, and consequently, many different substances exhibit the same behavior. This powerful notion of universality allows one to classify second-order phase transitions based on the underlying symmetries of the system without detailed knowledge of the microscopic dynamics. Some famous examples are the liquid-gas and ferromagnetic transitions, both of which possess critical points that are in the (static) universality class of the 3D Ising model. Universality identifies the singular behavior of the equation of state, say pressure as a function of temperature and chemical potential $p(T, \mu)$, with the Ising equation of state as a function of the Ising variables, i.e., the reduced temperature $r$ and the magnetic field $h$ near the critical point. However, neither the location of the critical point, nor the relation between $(T, \mu)$ and $(r, h)$ are universal, and for a quantitative description of the critical phenomena they have to be computed from the microscopic dynamics of the theory of interest. This is in many cases a mathematically intractable task, and often the solution can only be obtained as a series expansion at a point away from the critical point with a finite number of terms [1].

In this Letter, we consider the following general problem: given a truncated local series expansion of the equation of state in some parameter such as $\mu$, obtained away from the critical point, what can we say about the

Published by the American Physical Society under the terms of the Creative Commons Attribution 4.0 International license. Further distribution of this work must maintain attribution to the author(s) and the published article's title, journal citation, and DOI. Funded by SCOAP. critical behavior of the system? More precisely, how can we determine whether a critical point exists, and if it does, how can we reconstruct the singular behavior of the equation of state near the critical point from the truncated expansion obtained away from it? We show that it is possible to obtain a surprisingly large amount of information about the critical behavior of the system from the series coefficients, even if we have access to a modest number of them.

A major motivation for this work is the search for the conjectured critical point in the phase diagram of quantum chromodynamics (QCD) [2], which is one of the major outstanding problems in nuclear physics both theoretically and experimentally [3]. The theoretical approaches are severely limited by the sign problem that prevents firstprinciple lattice computations at nonzero baryon chemical potential $\mu_{B}$, and one of the methods to deal with this obstacle is to expand the equation of state around $\mu_{B}=0$ and compute the Taylor coefficients on the lattice without a sign problem (see [4] for a recent review). Another motivation is to understand the properties of strongly interacting fermions, such as unitary Fermi gases, where our theoretical knowledge of the equation of state is typically limited to the first few terms in the virial expansion [5].

Lee-Yang edge singularities.-Before detailing our method, we briefly summarize the Lee-Yang edge singularities that play a crucial role in our analysis. In their seminal work on phase transitions [6,7], Lee and Yang showed that the thermodynamic properties of a system is encoded in the distribution of the zeros of the partition function $Z(\zeta)$ as a function of fugacity, $\zeta=e^{\mu / T}$. For a finite system, the partition function is a positive polynomial for $\zeta \geq 0$. However, in general, it has zeros for complex values of $\mu$ and $T$. In the thermodynamic limit, the zeros coalesce into branch cuts emanating from the so-called 
Lee-Yang (LY) edge singularities. When the LY singularities pinch the real axis, the system exhibits a second-order phase transition. Likewise, the branch cut associated with a LY singularity crosses the real line when there is a firstorder phase transition.

The LY singularities are critical points themselves and have their own universality class. For example, for the Ising model, and $O(N)$ models in general, they are characterized by the $\phi^{3}$ theory with a pure imaginary coupling [8]. For these models, the analytical structure of the LY singularities has been studied $[9,10]$. In the complex plane of the scaling variable $x=h r^{-\beta \delta}$ [11], the singularities are located in the pure imaginary axis $x= \pm i x_{\mathrm{LY}}$, where $x_{\mathrm{LY}} \in \mathbb{R}$ has recently been calculated via the functional renormalization group method [13]. The magnetization around the LY singularity behaves as $m-m_{c} \sim\left(x \pm i x_{\mathrm{LY}}\right)^{\sigma}$ with the critical exponent $\sigma \approx 0.074-0.085$ for $d=3$ [14]. The LY singularities in the context of a QCD critical point has been discussed in, for example, [15-21].

Consider the equation of state of a theory near a critical point $\left(T_{c}, \mu_{c}\right)$ in the Ising universality class. From universality we can relate it to the Ising model via a linear map $[22,23]$

$$
\left(\begin{array}{l}
r \\
h
\end{array}\right):=\mathbb{M}\left(\begin{array}{l}
T-T_{c} \\
\mu-\mu_{c}
\end{array}\right)=\left(\begin{array}{ll}
r_{T} & r_{\mu} \\
h_{T} & h_{\mu}
\end{array}\right)\left(\begin{array}{l}
T-T_{c} \\
\mu-\mu_{c}
\end{array}\right) .
$$

This relation then leads to the following expression for the trajectory of the LY singularities [17]:

$$
\begin{aligned}
& \mu_{\mathrm{LY}}(T) \approx \mu_{c}-c_{1}\left(T-T_{c}\right) \pm i x_{\mathrm{LY}} c_{2}\left(T-T_{c}\right)^{\beta \delta}, \\
& \text { where } c_{1}:=\frac{h_{T}}{h_{\mu}} \quad c_{2}:=\frac{r_{\mu}{ }^{\beta \delta}}{h_{\mu}}\left(\frac{r_{T}}{r_{\mu}}-\frac{h_{T}}{h_{\mu}}\right)^{\beta \delta} .
\end{aligned}
$$

Notice that $c_{1}$ is the slope of the crossover line, whereas $c_{2}$ depends on the relative angle between the $h$ and $r$ axes $[22,23]$. Therefore, the trajectory in Eq. (2) depends on not only the location of the critical point, but also on the nonuniversal mapping parameters. We now explain how to optimally construct Eq. (2) from a series expansion.

The method.-Consider a thermodynamic function $f(T, \mu)$ (pressure, density, susceptibility, etc.) given a series expansion with a finite number of terms: $f(T, \mu) \sim$ $\sum_{n=0}^{N} f_{n}(T) \mu^{2 n}$. Our goal is to extract as much information from it as possible, especially about its singular behavior near a critical point if there is one. Obviously, in this form, what we have is a polynomial that does not exhibit any singular behavior. In principle, from a ratio test it is possible to determine the radius of convergence, which would indicate the location of the closest singularity to origin. However, when the nearest singularities are a complex conjugate pair of LY singularities (which is the case for a smooth crossover), the ratios of series coefficients do not converge monotonically, but rather have an oscillating envelope as a result of Darboux's theorem [24]. This makes numerically extracting the singularity from the ratios challenging. Alternatively, the singular behavior of $f(T, \mu)$ can be approximately constructed by a Padé resummation, $\mathrm{P}_{N / 2}[f]\left(\mu^{2}\right):=p\left(\mu^{2}\right) / q\left(\mu^{2}\right)$, where $p$ and $q$ are polynomials of order $N / 2$ whose the coefficients are determined by expanding $\mathrm{P}_{N / 2}[f]\left(\mu^{2}\right)$ and identifying the coefficients with the Taylor coefficients. The singularities of $f$, typically branch points, are approximated by the poles and zeros of the Padé approximant. However, as we will demonstrate later, Padé resummation has a known shortcoming: it creates spurious singularities which limits its range of applicability. In addition, it is also not the most optimal approximation scheme and can be dramatically improved by pairing with a conformal map [27]. The key idea is to apply an appropriately chosen conformal map $\mu^{2}:=\phi(z)$ and do the Padé resummation in $z: \mathrm{CP}_{N / 2}[f](z):=\tilde{p}(z) / \tilde{q}(z)$, where $\tilde{p}$ and $\tilde{q}$ are order $N / 2$ polynomials whose coefficients are determined by the Taylor coefficients of $f(\phi(z))$. The singularities of $\mathrm{CP}_{N / 2}[f](z)$ are mapped to the complex $\mu^{2}$ plane as via $\phi(z)$. For the remainder of this Letter, we shall refer to this method of extracting singularities simply as "conformal Padé." Unlike Padé, conformal Padé does not suffer from the spurious pole problems. It also provides an optimal approximation to the original function for a wide range of functions as proven in [27]. Our strategy is to first construct the trajectory (2) by extracting the $\mu_{\mathrm{LY}}(T)$ via conformal Padé for a sequence of temperatures and then to obtain the location of the critical point as well as the coefficients $c_{1}$ and $c_{2}$ from it. This is possible since the critical exponents are fixed by universality and, as mentioned, $x_{\mathrm{LY}}$ is known [13].

Conformal Padé methods are typically used to reconstruct Borel plane singularities in resumming asymptotic series, such as the $\epsilon$ expansion [28] or perturbation series for relativistic [29] and nonrelativistic [30] systems. Here we take a different approach and apply it to a convergent series to directly extract its singular behavior near the critical point. Our input, the series expansion of the equation of state, does not have to come from perturbation theory or the $\epsilon$ expansion. Our approach is similar to that of [1] but with a key differences: we focus on the complex LY singularities and conformal maps play a crucial role in reconstructing the equation of state.

The Gross-Neveu model.-To concretely demonstrate these ideas, we focus on the celebrated Gross-Neveu (GN) model [31], which is a four-fermion theory with the action

$$
S=\int d^{2} x\left(i \bar{\psi}\left(\not \partial-m_{q}\right) \bar{\psi}+\frac{g^{2}}{2}(\bar{\psi} \psi)^{2}\right),
$$

where $\psi$ is a Dirac fermion with $N_{f}$ flavors. It exhibits some of the key features of QCD, such as asymptotic freedom, chiral symmetry breaking, and dimensional 
transmutation. Notably, it was also shown that the GN model gives a reasonably good description of the firstorder phase transition in doped polyacetylene [32]. The theory has a discrete $\left(\mathbb{Z}_{2}\right)$ chiral symmetry $\psi \rightarrow \gamma_{5} \psi$ for $m_{q}=0$. We will work in the large $N_{f}$ limit $N_{f} \rightarrow \infty$ with $g^{2} N_{f}=$ fixed, where the fluctuations are suppressed and the mean field solution is exact.

The exact phase diagram of the GN model is known $[33,34]$ and has a rich structure such as spatially inhomogeneous kink-antikink crystals at high densities [34] and an exactly soluble, all-orders Ginzburg-Landau expansion [35]. However, in order to keep the discussion simple, we shall assume that the translational symmetry remains unbroken in this Letter. Furthermore, we focus on the crossover region of the phase diagram, which is not affected by the existence of inhomogeneous phases.

Thermodynamics of the model follows from minimizing the grand potential:

$$
\begin{aligned}
\Omega(\phi)= & \frac{\phi^{2}}{2 \pi}\left(\log \phi-\frac{1}{2}+\gamma\right)-\frac{\gamma}{\pi} \phi \\
& -T \int \frac{d k}{2 \pi} \prod_{\eta= \pm 1} \log \left[\left(1+e^{-\left(\sqrt{k^{2}+\phi^{2}}+\eta \mu\right) / T}\right)\right]
\end{aligned}
$$

with respect to $\phi$, which determines the fermion mass $M$ as a function of $T, \mu$, and $\gamma$. The parameter $\gamma \propto m_{q}$ is a measure of explicit chiral symmetry breaking and it vanishes in the chiral limit [33]. In our analysis, we will work with a fixed, nonzero value of $\gamma$. The equation of state is obtained by identifying the pressure as $p(T, \mu)=-\Omega[M(T, \mu)]:=-\min _{\phi} \Omega[\phi]$. The homogeneous phase diagram of the model is shown in Fig. 1. In the chiral limit $(\gamma=0)$, the ordered phase $M \neq 0$, where chiral

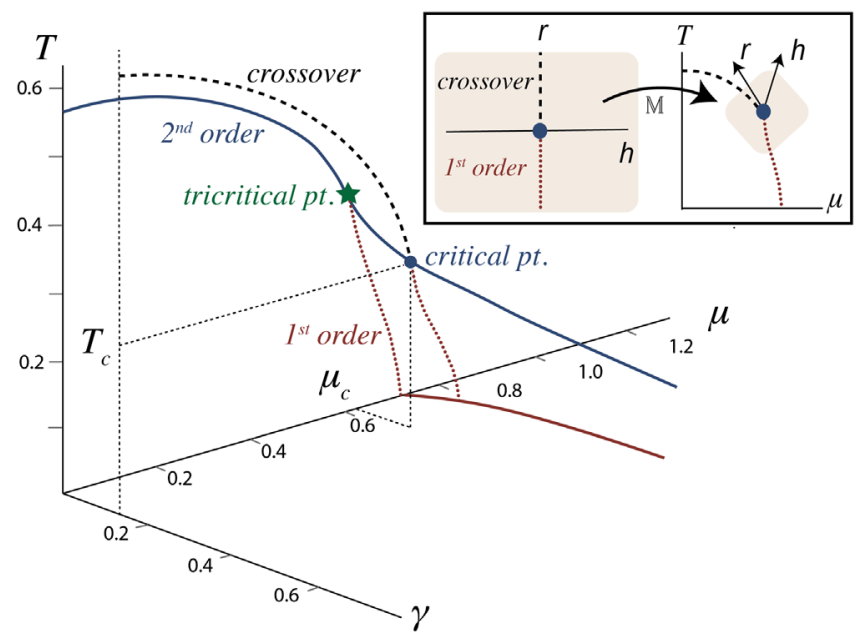

FIG. 1. The phase diagram of the Gross-Neveu model assuming unbroken translational symmetry. Inset: the mapping between the Ising model parameters, $r, h$ and $T, \mu$ near the critical point given in Eq. (1). symmetry is broken, and the disordered phase $M=0$, where the chiral symmetry is restored, are separated by a second(first)-order transition for $T<T_{\text {tc }}\left(T>T_{\text {tc }}\right)$ shown in solid blue (dotted red) curves. These curves merge at a tricritical point $\left(T_{\mathrm{tc}}, \mu_{\mathrm{tc}}\right) \approx(0.318,0.608)$ [36]. When the chiral symmetry is explicitly broken, for a fixed $\gamma \neq 0$, the transition is a smooth crossover for hight $T$ that ends at a critical point $\left(T_{c}, \mu_{c}\right)$ and turns into a first-order transition. Since the theory has a $\mathbb{Z}_{2}$ chiral symmetry, the second-order transition is in the same static universality class as the mean field Ising model. The trajectory of the LY singularities $\mu_{\mathrm{LY}}(T)$ is determined by the condition

$$
\partial_{\phi} \Omega(\phi)=\partial_{\phi}^{2} \Omega(\phi)=0 .
$$

In the crossover region $T>T_{c}$, this condition leads to a pair of complex solutions $\mu_{\mathrm{LY}}(T)=\operatorname{Re} \mu_{\mathrm{LY}}(T) \pm i \operatorname{Im} \mu_{\mathrm{LY}}(T)$, which coalesce and pinch the real axis at the ordinary critical point as expected: $\mu_{\mathrm{LY}}\left(T_{c}\right)=\mu_{c}$. In the vicinity of the critical point, $\mu_{\mathrm{LY}}(T)$ takes the scaling form given in Eq. (2) with the mean field exponents $\beta=1 / 2, \delta=3$,

$$
\mu_{\mathrm{LY}}(T) \sim \mu_{c}-c_{1}\left(T-T_{c}\right)+i c_{2} x_{\mathrm{LY}}\left(T-T_{c}\right)^{3 / 2},
$$

where in the mean field limit $x_{\mathrm{LY}}=2 / 3 \sqrt{3}$. In the next section, we compute $T_{c}, \mu_{c}, c_{1}$, and $c_{2}$ directly from a truncated series expansion of the equation of state and compare these results with the exact solution obtained by numerically solving Eq. (5).

Results.-We computed the equation of state perturbatively in $\mu^{2}$ by first solving $\partial_{\phi} \Omega(\phi)=0$ orderby-order for a range of temperatures with $\gamma=0.1$. By plugging this solution into Eq. (4) and expanding in $\mu^{2}$, we obtained the Taylor series expansion for the pressure $p(T, \mu) \approx \sum_{n=0}^{N} p_{2 n}(T) \mu^{2 n}$. To illustrate the numerical evaluation of the equation of state, we focus on the susceptibility,

$\chi(T, \mu)=\frac{\partial^{2} p}{\partial \mu^{2}} \approx \sum_{n=0}^{N-1}(2 n+2)(2 n+1) p_{2 n+2}(T) \mu^{2 n}$,

because its singular part in the vicinity of the critical point grows as $\chi(\mu) \sim \operatorname{Re}\left(\mu^{2}-\mu_{\mathrm{LY}}^{2}\right)^{\sigma-1}$, where $\sigma=1 / 2$ in the mean field limit. Of course, in many cases, it is very difficult to generate such large number of terms. Therefore, we also show results obtained by 11 terms for comparison. We computed the singularities both from Padé and conformal Padé, which are shown in Fig. 2 for two different temperatures very close to and away from the critical point. We used a conformal map, $\phi_{1}(z)=4 \mu_{\mathrm{LY}}^{2} z /(1+z)^{2}$, defined over a one-cut complex plane with a singularity located at $\mu_{\mathrm{LY}}^{2}$ to resolve $f$ near the singularity $\mu_{\mathrm{LY}}^{2}$. This map transforms a square-root branch into a simple pole and is numerically easy to evaluate [27]. Since we are dealing 


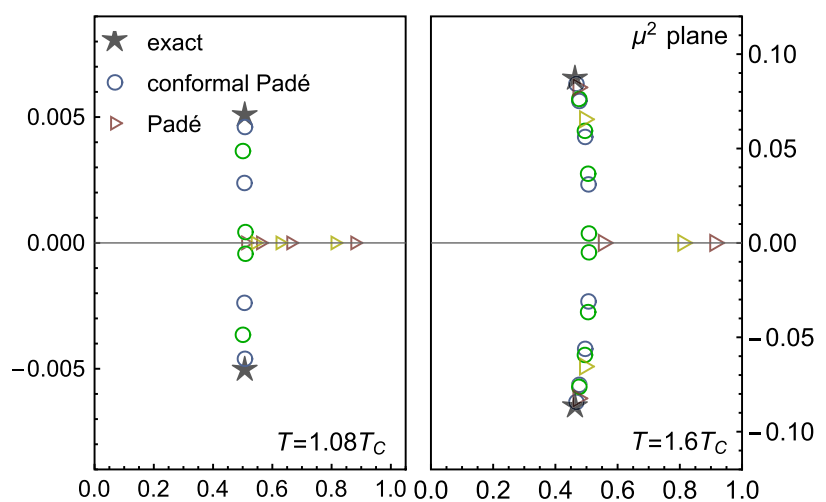

FIG. 2. The poles and zeros of Padé (red, yellow triangles, respectively) and conformal Padé (blue, green circles, respectively) approximants for two different temperatures compared with the exact locations of $\mu_{\mathrm{LY}}^{2}$.

with a two-cut plane with singularities located at $\mu_{\mathrm{LY}}^{2}$ and $\mu_{\mathrm{LY}}^{* 2}, \phi_{1}(z)$ does not eliminate the branch cut. Nevertheless it is fairly accurate in extracting location of the singularity locally. Since we do not know what $\mu_{\mathrm{LY}}$ is a priori, we first obtained a crude estimate for it from regular Padé, and we used it as an input in $\phi_{1}(z)$ and refined this estimate via conformal Padé.

In order to reconstruct the trajectory $\mu_{\mathrm{LY}}(T)$, we repeated this procedure for different temperatures. In order to smooth out the $T$ dependence of $\mu_{\mathrm{LY}}$ we used fits whose forms are determined from Eq. (6); namely, a linear fit for $\operatorname{Re} \mu_{\mathrm{LY}}(T)$ and a $y=a x^{3 / 2}$ fit for $\operatorname{Im} \mu_{\mathrm{LY}}(T)$. The results are shown in Fig. 3. From these fits we obtained the values of $T_{c}, \mu_{c}, c_{1}$, and $c_{2}$ shown in Table I.

Finally, we computed the susceptibility as a function $\mu$ via Padé and conformal Padé. In order to capture the global behavior of the equation of state, we used a different conformal map, $\phi_{2}(z)=4\left|\mu_{\mathrm{LY}}\right|^{2}\left[\theta /(1-z)^{2}\right] \theta[1-$ $\left.\theta /(1+z)^{2}\right]^{1-\theta}$ defined on a two-cut complex plane with two branch points located at $\left|\mu_{\mathrm{LY}}\right|^{2} e^{ \pm i \pi \theta}[27,29,30]$.

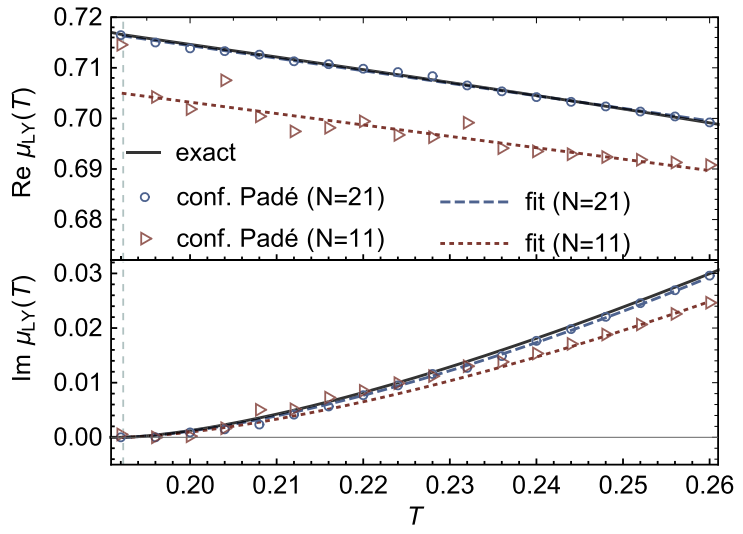

FIG. 3. The Lee-Yang singularity trajectory $\mu_{\mathrm{LY}}(T)$ reconstructed from conformal Padé with 20 and 10 terms. The vertical line denotes $T_{c}$.
TABLE I. The location of the critical point and the Ising model mapping parameters given in Eq. (6) extracted from conformal Padé.

\begin{tabular}{lcccc}
\hline \hline & $T_{c}$ & $\mu_{c}$ & $c_{1}$ & $c_{2}$ \\
\hline Exact & 0.192 & 0.717 & 0.249 & 4.684 \\
Conf. Padé $(N=21)$ & 0.195 & 0.716 & 0.248 & 4.323 \\
Conf. Padé $(N=11)$ & 0.185 & 0.707 & 0.225 & 3.666 \\
\hline \hline
\end{tabular}

The results for two representative temperatures near and away from $T_{c}$ are shown in Fig 4.

Discussion.-We now discuss these results. First, notice that, near the critical point $T=1.08 T_{c}$, Padé cannot resolve $\operatorname{Im} \mu_{\mathrm{LY}}$ and creates a sequence of poles and zeros along the real axis. Even when it does, away from the critical point $T=1.60 T_{c}$, there are still spurious poles along the real axis, which renders Padé (gray curves in Fig. 4) inapplicable for $\mu \gtrsim \operatorname{Re} \mu_{\mathrm{LY}}$. As seen from the same figures, conformal Padé does not suffer from such a problem and even for 11 terms, it does capture the qualitative behavior of the peak. For 21 terms, the agreement with the exact result up to $\mu \approx 0.8$ is quite remarkable. To refine the location of a given singularity $\mu_{\mathrm{LY}}$, we found the one-cut conformal map $\phi_{1}$ to be very accurate and easy to execute numerically. In order to capture the global properties of $\chi(T, \mu)$, we used a two-cut map $\phi_{2}$.

From the singularities extracted from conformal Padé, we constructed the LY singularity trajectory [Eq. (6)]. The critical temperature $T_{c}$ is obtained from the point $\operatorname{Im} \mu_{\mathrm{LY}}$

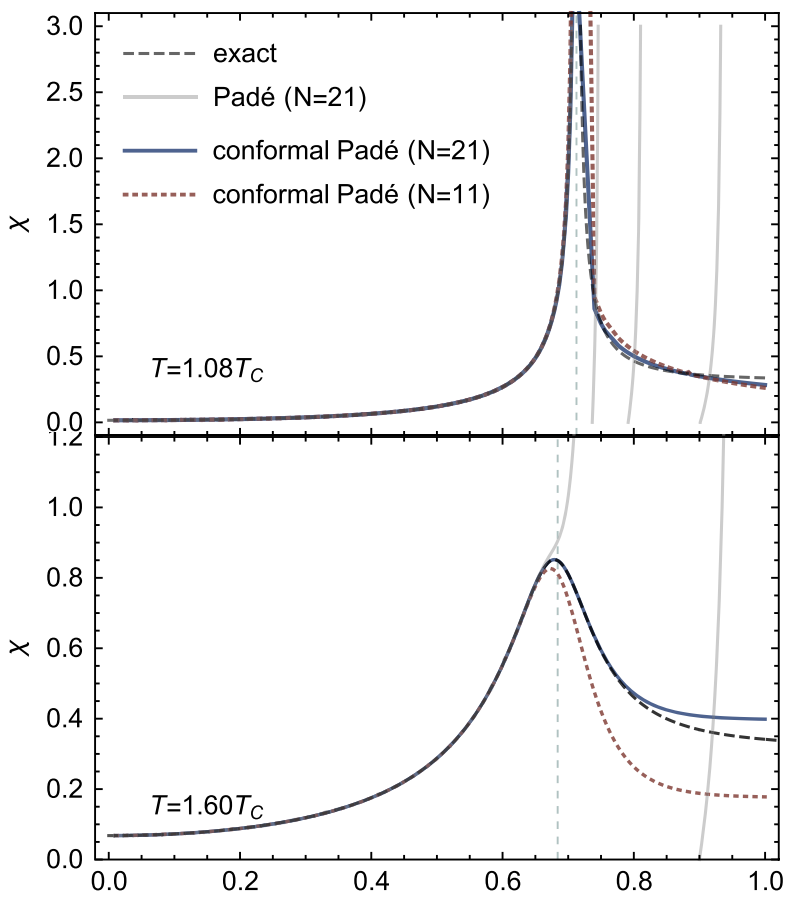

FIG. 4. The susceptibility as a function of $\mu$ for two different temperatures. Vertical lines denote $\operatorname{Re} \mu_{\mathrm{LY}}(T)$. 
vanishes (the vertical line in Fig. 3). Therefore, it is crucial to be able to accurately compute $\operatorname{Im} \mu_{\mathrm{LY}}$ where conformal Padé has a significant advantage over other methods, even with an input of 11 Taylor coefficients. The remaining parameters $\mu_{c}, c_{1}$, and $c_{2}$ are extracted from fits explained above. Notably, the location of the critical point can be determined with less than 5\% error even with 11 terms. The error is larger for the crossover slope, $c_{1}$ and $c_{2}$, however, it is below $10 \%$ for all cases, except for $c_{2}$ with 11 terms $(\approx 21 \%)$.

Summary and conclusions.-In this Letter, we tackled a general problem: given a truncated series expansion of the equation of state of a theory obtained away from any critical point, what can we say about a the existence of a critical point in the phase diagram and the singular behavior of the equation of state around it? We considered a fairly common case of a first-order transition that ends at a second-order critical point (which is in the Ising universality class) and turns into smooth crossover. We introduced a new way of extracting the leading singular behavior of the equation of state around the Lee-Yang edge singularity in the complex $\mu$ plane by using a combination of a conformal map and Padé resummation. Equipped with the knowledge of this trajectory, we showed how to extract the location of the critical point and to constrain the nonuniversal Ising mapping parameters in the scaling region. Finally, we demonstrated that this method significantly improves the numerical evaluation of the equation of state even with a modest number of Taylor coefficients.

There are various extensions and refinements of these ideas that we have left for future work. For example, one can extend this analysis to the first-order side $T<T_{c}$, where one expects the scaling part of the equation of state to jump to a different Riemann sheet $[9,37]$. This can be achieved by using the so-called "uniformization map" as a part of conformal Padé, which gives access to higher sheets.

An immediate application of the ideas we developed in this Letter is to assist the search for the critical point of QCD both by constraining its location and the Ising mapping parameters, as well as improving the numerical implementation of the equation of state in the hydrodynamic simulations. Based on the methods presented here and the Taylor coefficients calculated in [38], we preliminarily estimate that the location of the QCD critical point lies within the region $(T, \mu)=(141.0-146.1$, 501.8-662.7) $\mathrm{MeV}$. The interval partially takes into account the uncertainties in the coefficients due to the continuum extrapolation and statistics. One should interpret this estimate very cautiously. $T_{c}$ is expected to be lower than chiral transition temperature, which is estimated to be $T_{c}^{0}=132_{-6}^{+3} \mathrm{MeV}$ [39]. This discrepancy might be because the number of coefficients ( 4 as opposed to 11 ) is fairly low, the coefficients only go down to $T \approx 140 \mathrm{MeV}$, and have large uncertainties whose effects on conformal maps have not yet been fully understood. Therefore, although it is encouraging that this preliminary estimate is in the ballpark and consistent with those in $[19,38]$, moving forward, a detailed analysis of uncertainties in the Taylor coefficients is necessary. Likewise, other improvements, such as incorporating data from imaginary $\mu$ (e.g., [37]) within this framework, are left for future work.

We thank G. Dunne and M. Stephanov for valuable discussions. This work was supported by UNC Junior Faculty Development Award.

*gbasar@unc.edu

[1] M. E. Fisher, Critical point phenomena-the role of series expansions, Rocky Mt. J. Math. 4, 181 (1974).

[2] M. A. Stephanov, QCD phase diagram and the critical point, Prog. Theor. Phys. Suppl. 153, 139 (2004).

[3] A. Bzdak, S. Esumi, V. Koch, J. Liao, M. Stephanov, and N. $\mathrm{Xu}$, Mapping the phases of quantum chromodynamics with beam energy scan, Phys. Rep. 853, 1 (2020).

[4] C. Ratti, Lattice QCD and heavy ion collisions: A review of recent progress, Rep. Prog. Phys. 81, 084301 (2018).

[5] X.-J. Liu, Virial expansion for a strongly correlated Fermi system and its application to ultracold atomic Fermi gases, Phys. Rep. 524, 37 (2013).

[6] C.-N. Yang and T. D. Lee, Statistical theory of equations of state and phase transitions. 1. Theory of condensation, Phys. Rev. 87, 404 (1952).

[7] T. D. Lee and C.-N. Yang, Statistical theory of equations of state and phase transitions. 2. Lattice gas and Ising model, Phys. Rev. 87, 410 (1952).

[8] M.E. Fisher, Yang-Lee Edge Singularity and $\phi^{3}$ Field Theory, Phys. Rev. Lett. 40, 1610 (1978).

[9] X. An, D. Mesterházy, and M. A. Stephanov, On spinodal points and Lee-Yang edge singularities, J. Stat. Mech. (2018) 033207.

[10] X. An, D. Mesterhazy, and M. A. Stephanov, Critical fluctuations and complex spinodal points, Proc. Sci. CPOD2017 (2018) 040.

[11] $\beta$ and $\delta$ are the standard Ising critical exponents [12].

[12] J. Zinn-Justin, Quantum field theory and critical phenomena, Int. Ser. Monogr. Phys. 113, 1 (2002).

[13] A. Connelly, G. Johnson, F. Rennecke, and V. V. Skokov, Universal Location of the Yang-Lee Edge Singularity in $O(N)$ Theories, Phys. Rev. Lett. 125, 191602 (2020).

[14] X. An, D. Mesterházy, and M. A. Stephanov, Functional renormalization group approach to the Yang-Lee edge singularity, J. High Energy Phys. 07 (2016) 041.

[15] M. Halasz, A. Jackson, and J. Verbaarschot, Yang-Lee zeros of a random matrix model for QCD at finite density, Phys. Lett. B 395, 293 (1997).

[16] S. Ejiri, Lee-Yang zero analysis for the study of QCD phase structure, Phys. Rev. D 73, 054502 (2006),

[17] M. A. Stephanov, QCD critical point and complex chemical potential singularities, Phys. Rev. D 73, 094508 (2006).

[18] M. Wakayama, V. Bornyakov, D. Boyda, V. Goy, H. Iida, A. Molochkov, A. Nakamura, and V. Zakharov, Lee-Yang zeros in lattice QCD for searching phase transition points, Phys. Lett. B 793, 227 (2019). 
[19] S. Mukherjee and V. Skokov, Universality driven analytic structure of the QCD crossover: Radius of convergence in the baryon chemical potential, Phys. Rev. D 103, L071501 (2021).

[20] A. Connelly, G. Johnson, S. Mukherjee, and V. Skokov, Universality driven analytic structure of QCD crossover: Radius of convergence and QCD critical point, Nucl. Phys. A1005, 121834 (2021).

[21] C. Schmidt, J. Goswami, G. Nicotra, F. Ziesché, P. Dimopoulos, F. Di Renzo, S. Singh, and K. Zambello, Net-baryon number fluctuations, in Criticality in QCD and the Hadron Resonance Gas, arXiv:2101.02254.

[22] P. Parotto, M. Bluhm, D. Mroczek, M. Nahrgang, J. Noronha-Hostler, K. Rajagopal, C. Ratti, T. Schäfer, and M. Stephanov, QCD equation of state matched to lattice data and exhibiting a critical point singularity, Phys. Rev. C 101, 034901 (2020).

[23] M. S. Pradeep and M. Stephanov, Universality of the critical point mapping between Ising model and QCD at small quark mass, Phys. Rev. D 100, 056003 (2019).

[24] For some estimators, for the radius of convergence for QCD, see, i.e., $[25,26]$.

[25] M. Giordano and A. Pásztor, Reliable estimation of the radius of convergence in finite density QCD, Phys. Rev. D 99, 114510 (2019).

[26] M. Giordano, K. Kapas, S. Katz, D. Nogradi, and A. Pasztor, Towards a reliable lower bound on the location of the critical endpoint, Nucl. Phys. A1005, 121986 (2021).

[27] O. Costin and G. V. Dunne, Uniformization and constructive analytic continuation of Taylor series, arXiv:2009.01962.

[28] R. Guida and J. Zinn-Justin, Critical exponents of the $N$ vector model, J. Phys. A 31, 8103 (1998).

[29] M. Serone, G. Spada, and G. Villadoro, $\lambda \phi_{2}^{4}$ theory-Part II. The broken phase beyond NNNN(NNNN)LO, J. High Energy Phys. 05 (2019) 047.

[30] R. Rossi, T. Ohgoe, K. Van Houcke, and F. Werner, Resummation of Diagrammatic Series with Zero
Convergence Radius for Strongly Correlated Fermions, Phys. Rev. Lett. 121, 130405 (2018).

[31] D. J. Gross and A. Neveu, Dynamical symmetry breaking in asymptotically free field theories, Phys. Rev. D 10, 3235 (1974).

[32] A. Chodos and H. Minakata, The Gross-Neveu model as an effective theory for polyacetylene, Phys. Lett. A 191, 39 (1994).

[33] O. Schnetz, M. Thies, and K. Urlichs, Phase diagram of the Gross-Neveu model: Exact results and condensed matter precursors, Ann. Phys. (Amsterdam) 314, 425 (2004).

[34] O. Schnetz, M. Thies, and K. Urlichs, Full phase diagram of the massive Gross-Neveu model, Ann. Phys. (Amsterdam) 321, 2604 (2006).

[35] G. Basar, G. V. Dunne, and M. Thies, Inhomogeneous condensates in the thermodynamics of the chiral NJL(2) model, Phys. Rev. D 79, 105012 (2009).

[36] All dimensionful quantities in this Letter, such as $T$ and $\mu$, are expressed in units of the vacuum fermion mass.

[37] P. Fonseca and A. Zamolodchikov, Ising field theory in a magnetic field: Analytic properties of the free energy, arXiv: hep-th/0112167.

[38] A. Bazavov, H. T. Ding, P. Hegde, O. Kaczmarek et al., The QCD equation of state to $\mathcal{O}\left(\mu_{B}^{6}\right)$ from lattice QCD, Phys. Rev. D 95, 054504 (2017).

[39] H.-T. Ding, P. Hegde, O. Kaczmarek, F. Karsch, A. Lahiri, S.-T. Li, S. Mukherjee, H. Ohno, P. Petreczky, C. Schmidt, and P. Steinbrecher (HotQCD Collaboration), Chiral Phase Transition Temperature in $(2+1)$-Flavor QCD, Phys. Rev. Lett. 123, 062002 (2019).

[40] S. Borsányi, Z. Fodor, J. N. Guenther, R. Kara, S. D. Katz, P. Parotto, A. Pásztor, C. Ratti, and K. K. Szabó, Lattice QCD Equation of State at Finite Chemical Potential from an Alternative Expansion Scheme, Phys. Rev. Lett. 126, 232001 (2021). 